\title{
Factors influencing pore-pressure prediction in complex carbonates based on effective medium theory
}

\author{
Wang Ruihe*, Wang Zizhen, Shan Xun, Qiu Hao and Li Tianyang \\ School of Petroleum Engineering, China University of Petroleum, Qingdao, Shandong 266580, China \\ (C) China University of Petroleum (Beijing) and Springer-Verlag Berlin Heidelberg 2013
}

\begin{abstract}
A calculation model based on effective medium theory has been developed for predicting elastic properties of dry carbonates with complex pore structures by integrating the Kuster-Toksǒz model with a differential method. All types of pores are simultaneously introduced to the composite during the differential iteration process according to the ratio of their volume fractions. Based on this model, the effects of pore structures on predicted pore-pressure in carbonates were analyzed. Calculation results indicate that cracks with low pore aspect ratios lead to pore-pressure overestimation which results in lost circulation and reservoir damage. However, moldic pores and vugs with high pore aspect ratios lead to pore-pressure underestimation which results in well kick and even blowout. The pore-pressure deviation due to cracks and moldic pores increases with an increase in porosity. For carbonates with complex pore structures, adopting conventional pore-pressure prediction methods and casing program designs will expose the well drilling engineering to high uncertainties. Velocity prediction models considering the influence of pore structure need to be built to improve the reliability and accuracy of pore-pressure prediction in carbonates.
\end{abstract}

Key words: Carbonates, effective medium theory, elastic properties, pore-pressure prediction, pore structure

\section{Introduction}

The mechanism of abnormal pore-pressure in carbonates is complicated. It is not the same as the under compaction mechanism of clastic sedimentary rocks. Based on the compaction mechanism, either explicitly or implicitly, the relation of velocity-porosity-effective stress is usually employed to predict the pore-pressure in oil and gas exploration (Dutta, 2002). Although these conventional methods are not appropriate for pore-pressure prediction in carbonates due to abnormal pore-pressure mechanisms, they are still widely used in engineering practice. The accuracy of the velocity-porosity relationship directly affects the reliability of the predicted pore-pressure (Chopra and Huffman, 2006; Gutierrez et al, 2006; Zhang and Wieseneck, 2011).

The elastic wave velocity in underground rocks is an important indicator of abnormal pore-pressure. The velocities in porous rocks are influenced by many factors, such as the mineral composition, porosity, pore fluids, pore structure, effective stress, and temperature (Chen et al, 2009; Wang,

*Corresponding author. email: wangrh@upc.edu.cn Received June 15, 2013
2001). Because of dissolution and dolomitization in the diagenetic process, secondary pores are well developed and the pore structure is extremely complicated in carbonates (Ma et al, 1999). Therefore, an empirical velocity-porosity relationship used for sandstones may lead to a large deviation and even erroneous results when applied for carbonates. Experimental studies indicate that the velocity-porosity relationship of carbonates is scattered due to the complex pore structures of different carbonates (Kenter and Ivanov, 1995; Anselmetti et al, 1997; Wang, 2002; Assefa et al, 2003; Weger, 2006; Verwer et al, 2008). Fig. 1 shows the relationship between P-wave velocity and porosity in carbonates based on logging data from three wells. As can be seen from Fig. 1, the difference of P-wave velocity reaches $3,631 \mathrm{~m} / \mathrm{s}$ under the same porosity, while the difference in porosity reaches $14.7 \%$ with the same P-wave velocity. Eberli et al (2003) pointed out that the pore structure of carbonates is nearly equally important to porosity in their elastic behavior and resultant acoustic velocity. Scatter of velocity-porosity relationships in carbonates increases the uncertainty of porepressure prediction using conventional methods, which is the main difficulty in predicting the pore-pressure in carbonates.

The elastic wave velocity is an objective reflection of the rock elasticity. Different pore structures with the same 
porosity have different pore stiffness, which is the internal mechanism of velocity scattering in carbonates. In this paper, a model of elastic properties for dry carbonates with complex pore structures is developed by combining the classic Kuster-Toksǒz model (KT model) with a differential method based on the effective medium theory, and this model is named KTDEM model. Subsequently the elastic velocities are calculated, and the influences of pore structures on pore -pressure prediction are analyzed.

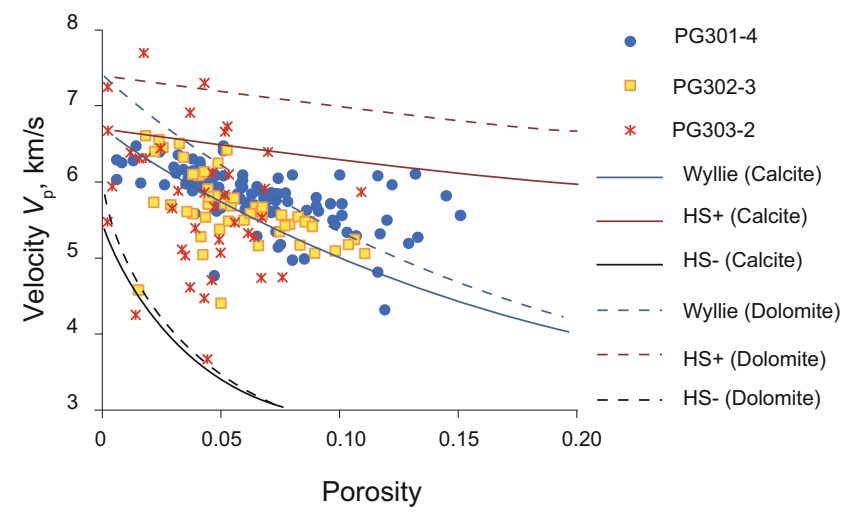

Fig. 1 Wide scattering of velocity-porosity relationship in carbonates

\section{Elastic properties of carbonates with complex pore structures}

\subsection{Rock physics model for carbonates}

Inspired by the construction methodology of the Xu-White model (Xu and White, 1995), the KT model is integrated with the differential method to describe the elastic properties of carbonates with complex pore structures. Fig. 2 shows the flowchart of integrating the KT model with the differential method.

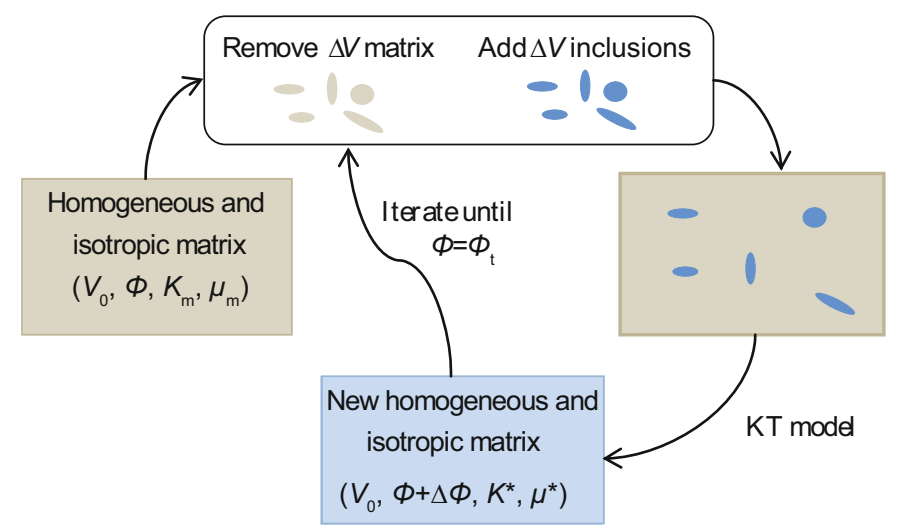

Fig. 2 The flowchart of integrating KT model with a differential method

Based on long-wavelength first-order scattering theory, Kuster and Toksǒz (1974) developed a model to estimate the elastic properties of porous materials. It also considered the effects of pore shape. By setting $M=(K, \mu), T=(P, Q)$, the generalized expression of the KT model is as follows (Liu, 2013),

$$
\left(\boldsymbol{M}-\boldsymbol{M}_{\mathrm{m}}\right) \frac{\boldsymbol{M}_{\mathrm{m}}+\frac{4}{3} \boldsymbol{R}_{\mathrm{m}}}{\boldsymbol{M}+\frac{4}{3} \boldsymbol{R}_{\mathrm{m}}}=\sum_{i=1}^{N} x_{i}\left(\boldsymbol{M}_{i}-\boldsymbol{M}_{\mathrm{m}}\right) \boldsymbol{T}_{\mathrm{m} i}
$$

with

$$
\begin{aligned}
& \boldsymbol{R}_{\mathrm{m}}=\left(\mu_{\mathrm{m}}, \frac{\mu_{\mathrm{m}}\left(9 K_{\mathrm{m}}+8 \mu_{\mathrm{m}}\right)}{8\left(K_{\mathrm{m}}+2 \mu_{\mathrm{m}}\right)}\right) \\
& \boldsymbol{M}_{\mathrm{m}}=\left(K_{\mathrm{m}}, \mu_{\mathrm{m}}\right) \\
& \boldsymbol{M}_{i}=\left(K_{i}, \mu_{i}\right) \\
& \boldsymbol{T}_{\mathrm{m} i}=\left(P_{\mathrm{m} i}, Q_{\mathrm{m} i}\right)
\end{aligned}
$$

where $K$ and $\mu$ are to-be-determined effective bulk and shear moduli of the porous rock, respectively; $K_{\mathrm{m}}$ and $\mu_{\mathrm{m}}$ are the bulk and shear moduli of the rock matrix, respectively; $K_{i}$ and $\mu_{i}$ are the bulk and shear moduli of the $i$ th inclusion, respectively; $x_{i}$ is the volume fraction of the $i$ th inclusion, $P_{\mathrm{m} i}$ and $Q_{\mathrm{m} i}$ are the geometrical factors for the $i$ th inclusion that depend on the aspect ratio $\alpha$ ( $\alpha$ is the ratio of short axis to long axis). The calculation method of $P_{\mathrm{m} i}$ and $Q_{\mathrm{m} i}$ can refer to Kuster and Toksǒz (1974). The KT model is limited to be used in a dilute concentration $(\phi / \alpha<<1)$ of pores and ultrasonic laboratory conditions (Xu and White, 1995) because the pores are isolated with respect to flow.

Fig. 3 shows the differential iteration process described by the path change of porosity. For rocks with two types of pores, the porosity of the rock is assumed to reach a point $A\left(\phi_{1 k}, \phi_{2 k}\right)$ after $k$ steps of iteration along a random path. The volume fractions of any type of pores monotonically increase through the iteration process. Therefore, any path from $A$ to $B C D$ within the dashed area can be applied for the $(k+1)$ th iteration. And if the added-volume ratio of these two types of pores are changed at each iteration step, there will be numerous paths to complete the whole differential iteration process from the original point $O(0,0)$ to the terminal point $T\left(\phi_{1 t}, \phi_{2 t}\right)$. Elastic moduli of dry rocks do not depend on porosity-path significantly (Kumar and Han, 2005), therefore it can significantly simplify the differential iteration process

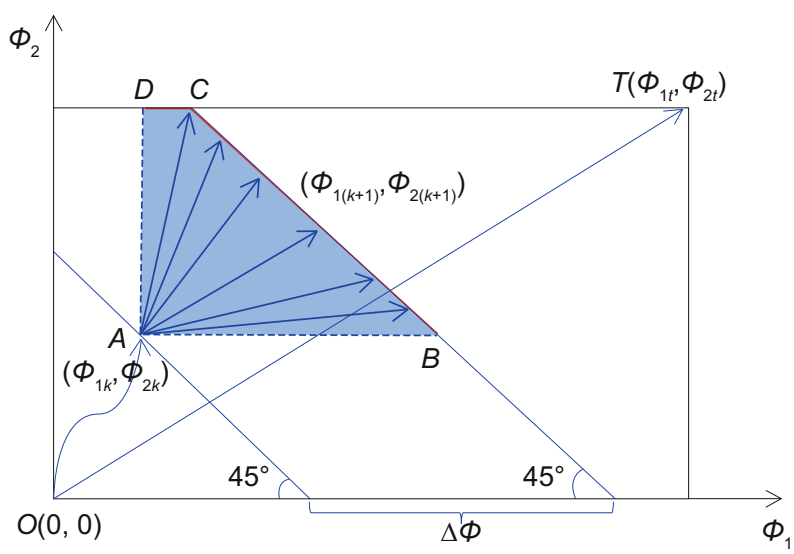

Fig. 3 Differential iteration process by changing the path of porosity 
to follow the path of straight line $O T$, i.e., all kinds of pores are simultaneously added according to the ratio of their volume fractions.

In a general scenario, rocks with volume of $V_{0}$ have $N$ types of pores. The porosity is

$$
\phi_{\mathrm{t}}=\sum_{i=1}^{N} \phi_{i}
$$

where $\phi_{\mathrm{t}}$ is the desired total porosity, and $\phi_{i}$ is the desired porosity of the $i$ th type of pores.

The volume of the $i$ th type of pores is $V_{0} \phi_{i k}$ after the $k$ th iteration and it is $V_{0}\left(\phi_{i k}+\Delta \phi_{i}\right)$ after the $(k+1)$ th iteration. For the $(k+1)$ th iteration, the effective matrix with volume $\Delta V$ is firstly removed among which the volume of the $i$ th type of pores is $\Delta V \phi_{i k}$. And then pores with volume $\Delta V$ are added among which the volume of the $i$ th type of pores is $\Delta V \frac{\phi_{i}}{\phi_{\mathrm{t}}}$. According to the conservation of volume, the following relation can be deduced,

$$
V_{0} \phi_{i k}-\Delta V \phi_{i k}+\Delta V \frac{\phi_{i}}{\phi_{\mathrm{t}}}=V_{0}\left(\phi_{i k}+\Delta \phi_{i}\right)
$$

The volume fraction of the $i$ th type of pores in the $(k+1)$ th iteration is

$$
x_{i}=\frac{\Delta V}{V_{0}} \frac{\phi_{i}}{\phi_{\mathrm{t}}}
$$

Because all kinds of pores are simultaneously added to the composite according to the ratio of their volume fractions, the following form can be given,

$$
\frac{\Delta \phi_{i}}{\Delta \phi}=\frac{\phi_{i k}}{\phi}=\frac{\phi_{i}}{\phi_{\mathrm{t}}}
$$

By substituting Eq. (5) into Eq. (3) and rearrangement, Eq. (4) can be rewritten as,

$$
x_{i}=\frac{\phi_{i}}{\phi_{\mathrm{t}}} \frac{\Delta \phi}{1-\phi}
$$

Pores in carbonates can be classified into three sets based on their shapes and stiffness (Kumar and Han, 2005; Xu et al, 2007): 1) cracks and fractures (CF), which have pore aspect ratios smaller than 0.02 and tend to deform under compaction; 2) moldic pores and vugs (MV), which have pore aspect ratios larger than 0.6 and higher stiffness to resist deformation; and 3 ) interparticle and intercrystalline pores (IP), which have pore aspect ratios ranging from 0.1 to 0.3 and moderate stiffness. During the differential iteration process, the KT model can be written as Eq. (7) by substituting Eq. (6) into Eq. (1),

$$
\begin{aligned}
& {[\boldsymbol{M}(\phi+\Delta \phi)-\boldsymbol{M}(\phi)] \frac{\left[\boldsymbol{M}(\phi)+\frac{4}{3} \boldsymbol{R}(\phi)\right]}{\left[\boldsymbol{M}(\phi+\Delta \phi)+\frac{4}{3} \boldsymbol{R}(\phi)\right]}} \\
& =\sum_{i} \frac{\phi_{i}}{\phi_{\mathrm{t}}} \frac{\Delta \phi}{1-\phi}\left[\boldsymbol{M}_{i}-\boldsymbol{M}(\phi)\right] \boldsymbol{T}_{i}(\phi) \quad(i=\mathrm{IP}, \mathrm{CF}, \mathrm{MV})
\end{aligned}
$$

where $\phi_{\mathrm{IP}}, \phi_{\mathrm{CF}}$ and $\phi_{\mathrm{MV}}$ represent interparticle porosity, crack porosity and moldic porosity, respectively; $\boldsymbol{M}(\phi)=(K(\phi), \mu(\phi)), K(\phi)$ and $\mu(\phi)$ are the effective bulk and shear moduli with respect to $\phi$. Because $\Delta \phi$ is very small, the fractional term $\frac{\boldsymbol{M}(\phi)+\frac{4}{3} \boldsymbol{R}(\phi)}{\boldsymbol{M}(\phi+\Delta \phi)+\frac{4}{3} \boldsymbol{R}(\phi)}$ on the lefthand side of Eq. (7) is nearly equal to 1. Therefore, Eq. (7) can be rewritten simply with satisfactory accuracy,

$$
\begin{aligned}
\boldsymbol{M}(\phi+\Delta \phi)-\boldsymbol{M}(\phi) & =\sum_{i} \frac{\phi_{i}}{\phi_{\mathrm{t}}} \frac{\Delta \phi}{1-\phi}\left[\boldsymbol{M}_{i}-\boldsymbol{M}(\phi)\right] \boldsymbol{T}_{i}(\phi) \\
(i & =\mathrm{IP}, \mathrm{CF}, \mathrm{MV})
\end{aligned}
$$

When performing Eq. (8), the added porosity at each step of the iteration should meet the "dilute concentration" requirement of the KT model. This method is able to calculate the elastic properties of dry carbonates with different pore structures and porosities through certain steps of iteration. Then the effect of water saturation can be considered by Gassmann's equation (Gassmann, 1951), and corresponding elastic wave velocities can be calculated. The results can provide basic data for analyzing the pore-pressure deviation.

\subsection{Model verification}

In order to verify the reliability of the model (KTDEM) described in Eq. (8), the calculation results are compared with those obtained from both the classic KT model and the DEM model (Berryman, 1992). The parameters used in the calculation are shown in Table 1, and the results are shown in Fig. 4.

Table 1 Parameters used for calculation

\begin{tabular}{cc}
\hline Composition & Parameters \\
\hline Rock matrix & $K_{\mathrm{m}}=76.8 \mathrm{GPa}$ \\
(calcite) & $\begin{array}{c}\mu_{\mathrm{m}}=32.0 \mathrm{GPa} \\
\rho_{\mathrm{m}}=2.71 \mathrm{~g} / \mathrm{cm}^{3}\end{array}$ \\
\hline Pore fluids & $K_{\mathrm{f}}=0 \mathrm{GPa}$ \\
(air) & $\begin{array}{c}\mu_{\mathrm{f}}=0 \mathrm{GPa} \\
\rho_{\mathrm{f}}=0 \mathrm{~g} / \mathrm{cm}^{3}\end{array}$ \\
\hline Pore structure & $\phi_{\mathrm{MV}}=20 \% \phi, \alpha_{\mathrm{MV}}=0.8$ \\
$\phi_{\mathrm{IP}}=80 \% \phi, \alpha_{\mathrm{IP}}=0.1$
\end{tabular}

According to the applicable conditions and given parameters, the dilute concentration of pores required by the $\mathrm{KT}$ model is $\phi \leq 0.1$ in this case. The results of the KT model are accurate and reliable within this low-porosity range. As shown in Fig. 4, when $\phi \leq 0.1$, the result of the KTDEM model is close to that of the KT model, the average deviation of bulk modulus is $0.898 \%$ with a maximum deviation of $3.35 \%$, and the average deviation of shear modulus reaches $0.882 \%$ with a maximum deviation of $2.50 \%$. When $\phi>0.1$, the bulk modulus calculated by the KT model show a large deviation irrationally. The result of the KTDEM model is consistent with that of the DEM model and has satisfactory accuracy 


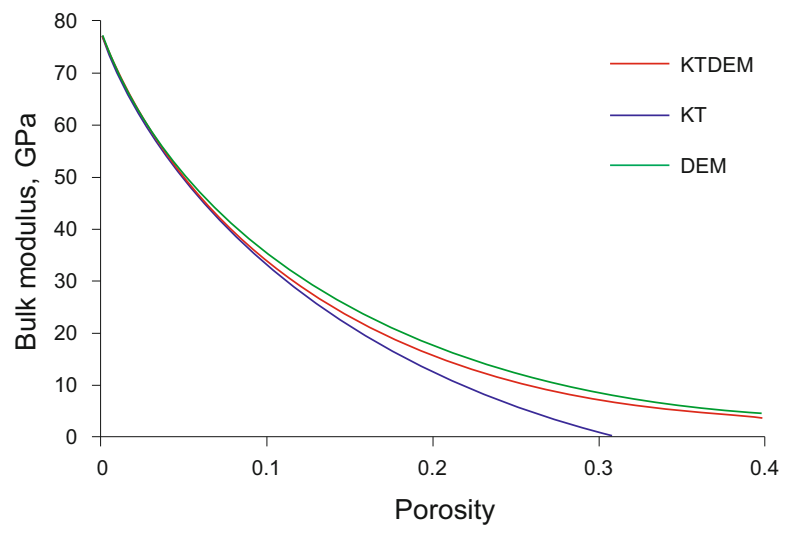

(a) Bulk modulus

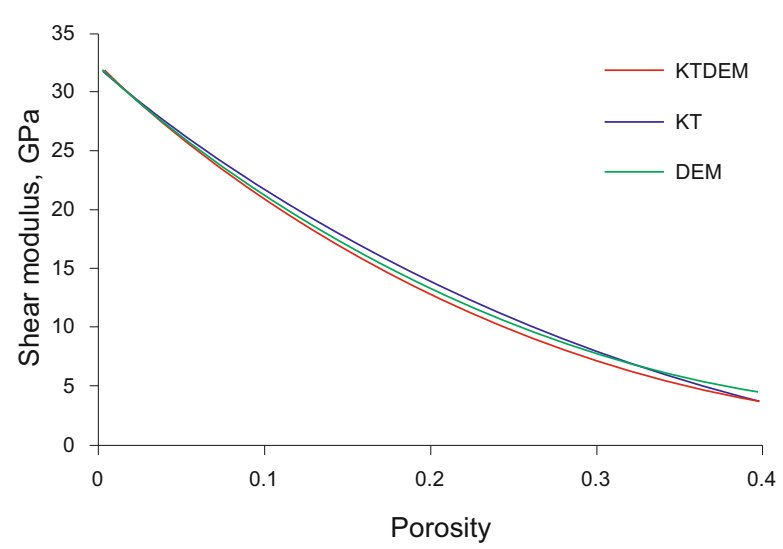

(b) Shear modulus

Fig. 4 Comparison of poroelastic properties calculated by the KTDEM, KT and DEM models

within the whole porosity range. Compared with the DEM model, the average deviation of the bulk modulus calculated by the KTDEM model is $5.22 \%$, and the average deviation of shear modulus is $3.74 \%$. The resultant average deviation of the P-wave velocity in dry carbonates is only $2.27 \%$. When $\phi<0.2$, the maximum deviations of both bulk and shear moduli are less than $4.5 \%$, the resultant maximum deviation of P-wave velocity is less than $1.8 \%$, which indicates the robust reliability of the KTDEM model. However, the DEM model needs to introduce different type of pores one after another (Sun et al, 2012) for rocks with more than two types of pores, which is complicated to conduct and has a heavy calculation burden. The KTDEM model proposed in this paper simultaneously adds all types of pores to the composite according to the ratio of their volume fractions, which is much easier to realize and needs less calculation.

\section{Analysis of factors influencing pore- pressure prediction}

\subsection{Deviation of pore-pressure prediction}

The mechanism of abnormal pore-pressure in carbonates is different from the undercompaction mechanism of clastic sedimentary rocks. However, no attempts have been made to consider this in pore-pressure prediction models and no methods have been developed specially for carbonate reservoirs, and the models based on the compaction mechanism are still widely used. If the effects of pore structure on the velocity-porosity relationship are considered, the conventional methods may lead to a large deviation for pore-pressure prediction. For a carbonate reservoir with a normal pore-pressure, it is assumed that its depth is $h$, porosity is $\phi$ and corresponding velocity under normal compaction is $v_{\mathrm{n}}$. While its actual velocity is $v$ due to the effects of pore structure. $\Delta \rho=\frac{p_{\mathrm{p}}-p_{\mathrm{w}}}{g h}$ is the equivalent density of pore-pressure deviation, which represents the deviation between the predicted pore-pressure and the normal pressure. Applying Eaton's model gives,

$$
\Delta \rho_{\mathrm{p}}=\left(\bar{\rho}-\rho_{\mathrm{w}}\right)\left[1-\left(\frac{v}{v_{\mathrm{n}}}\right)^{n}\right]
$$

where $v_{\mathrm{n}}$ can be calculated by Wyllie's time average equation, and the average density of formation $\bar{\rho}$ can be calculated by the following empirical relations (Sayers et al, 2002; Traugott, 1997),

$$
\bar{\rho}(h)=1.9532+0.1198\left(\frac{v_{\mathrm{n}}-v_{0}}{952.5 k}\right)^{0.6}
$$

The empirical parameters in Eq. (10) can be determined according to the optimized results (Gutierrez et al, 2006; Xu et al, 1993; Sayers et al, 2006) based on data from the Gulf of Mexico: $n=2.3, k=0.6 \mathrm{~s}^{-1}, v_{0}=1584.96 \mathrm{~m} / \mathrm{s}$. By using P-wave velocities of carbonates for different pore structures, the corresponding equivalent density of pore-pressure deviation can be calculated from Eq. (9).

\subsection{Influence of pore aspect ratio}

Fig. 5 shows the relationship between pore-pressure deviation and pore aspect ratio with only one type of pore in carbonates. The equivalent density of pore-pressure deviation changes from positive to negative with an increase in pore

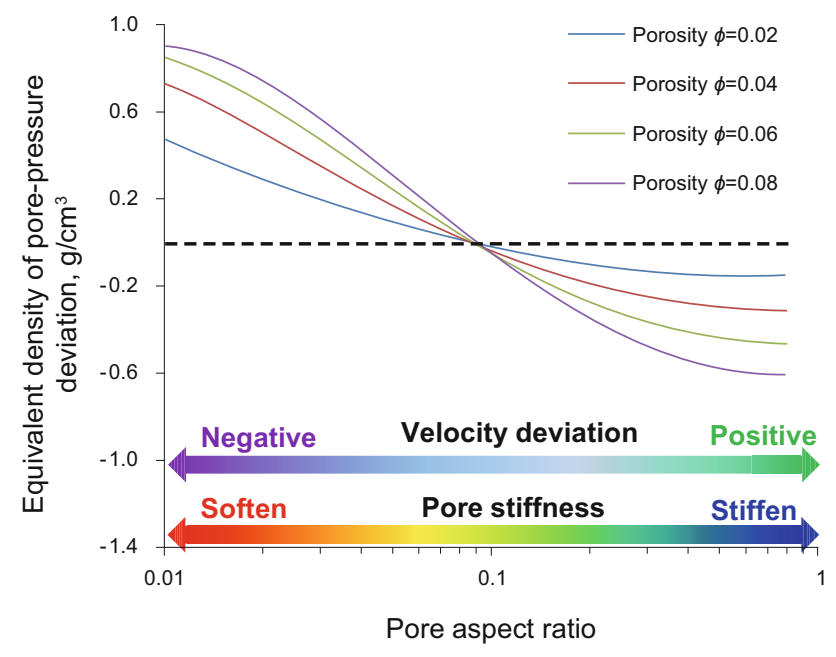

Fig. 5 Relationship between pore-pressure deviation and pore aspect ratio in carbonates 
aspect ratio. Cracks with lower pore aspect ratio lead to porepressure overestimation and the equivalent density of porepressure deviation is positive. However, moldic pores and vugs with higher pore aspect ratios lead to pore-pressure underestimation and the equivalent density of pore-pressure deviation is negative. When the pore aspect ratio is close to 0.1 , the deviation of pore-pressure is around zero. The dominant pore type in carbonates is interparticle pores under this condition and Wyllie's time average equation can give reliable description of the velocity-porosity relationship.

Pores with different pore aspect ratios have different pore stiffness (Fig. 5), and lead to different P-wave velocities, which is the underlying reason for pore-pressure deviation. The analysis of experimental and logging data (Anselmetti and Eberli, 1999; Xu et al, 2007; Wang et al, 2011) shown that taking the velocity of rocks with all interparticle pores as reference, the velocity becomes smaller with the increase in soft cracks and results in a negative velocity deviation. On the contrary, the velocity becomes greater with the increase in stiff moldic pores or vugs and leads to a positive velocity deviation. Only completely considering the influence of different types of pores in the velocity prediction model can accurately predict pore-pressure in carbonates.

\subsection{Influence of porosity}

The deviations of predicted pore-pressure are calculated under different porosities of cracks, interparticle pores and moldic pores respectively. The aspect ratio of cracks, interparticle pores and moldic pores are $0.02,0.1$ and 0.8 , respectively. Fig. 6 shows the effect of porosity on porepressure deviation in carbonates.

As can be seen from Fig. 6, the pore-pressure deviation caused by interparticle pores is close to zero, which shows that the pore-pressure deviation is independent of porosity. The pore-pressure deviation caused by cracks and moldic pores increases with an increase in porosity. When the volume of cracks increases to some extent, the positive pore-pressure deviation caused by cracks tends to be stable. That is because the softening effect of cracks is no longer enhanced and the effective elastic moduli reach the Hashin-Shtrikman lower bound under this condition.

The deviation of pore-pressure caused by cracks is much larger than that of moldic pores for common pore structures

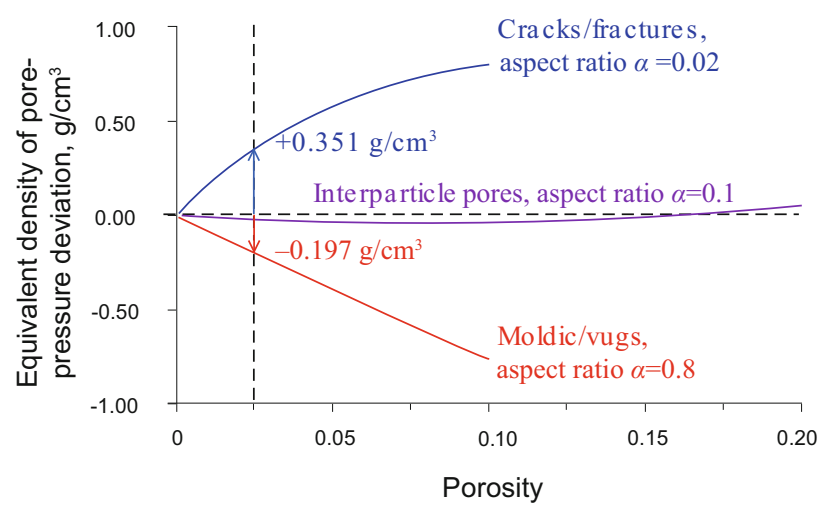

Fig. 6 Effect of porosity on pore-pressure deviation in carbonates of carbonates. As shown in Fig. 6, when the porosity is $2.5 \%$, the pore-pressure deviation caused by cracks is $+0.351 \mathrm{~g} /$ $\mathrm{cm}^{3}$. For the same volume of moldic pores or vugs, the corresponding pore-pressure deviation is about $-0.197 \mathrm{~g} /$ $\mathrm{cm}^{3}$. However, the equivalent density of safety margin for formation fracture pressure is only $0.03 \mathrm{~g} / \mathrm{cm}^{3}$ and the safety margin for kick is $0.05-0.10 \mathrm{~g} / \mathrm{cm}^{3}$ according to the design method of casing program (SY/T 5431-2008). Therefore, if the design of drilling engineering does not consider the influence of pore structure, problems such as lost circulation and formation damage can easily occur in crack-enriched layers due to the pore-pressure overestimation. In formations where moldic pores or vugs are well developed, the well is more likely to encounter well kick and even blowout because of the pore-pressure underestimation.

Overall, because of the influence of pore structure, it will expose well drilling engineering to high uncertainties if conventional pore-pressure prediction methods and casing program designs are adopted for carbonate reservoirs. Based on the characteristics of pore structures in carbonates, it can improve the reliability and accuracy of pore-pressure prediction in carbonates, as well as decrease the risk of drilling engineering to quantify the influence of pore structure e.g., porosity and pore aspect ratio, on the elastic wave properties.

\section{Conclusions}

1) A calculation model for elastic properties of dry carbonates with complex pore structures has been developed by integrating the KT model and the differential method. All types of pores are simultaneously introduced into the composite according to the ratio of their volume fractions during the differential iteration process. The model is verified to be reliable.

2) Using conventional methods to predict pore-pressure in carbonates may bring large deviations due to the complex pore structure. The deviation of pore-pressure is affected by both porosity and pore aspect ratio. Cracks with low pore aspect ratios lead to a positive pore-pressure deviation. Moldic pores and vugs with high pore aspect ratios lead to a negative pore-pressure deviation. The pore-pressure deviation caused by cracks and moldic pores increased with an increase in porosity. The pore-pressure deviation caused by interparticle pores is close to zero, which does not show a dependence on porosity.

3) The pore-pressure is overestimated in crackenriched layers, which tends to induce problems such as lost circulation and formation damage. The pore-pressure is underestimated in formations where the moldic pores or vugs are well developed, which is more likely to result in well kick and even blowouts. The influence of pore structure in carbonate reservoirs can expose the well drilling engineering to higher security risks when adopting conventional porepressure prediction methods and/or casing program design. It is necessary to develop a velocity prediction model which considers the influence of pore structure since it can improve the reliability and accuracy of pore-pressure prediction in carbonates. 


\section{Acknowledgements}

The authors acknowledge the financial support from the National Natural Science Foundation of China (No. 51274230), the Natural Science Foundation of Shandong Province (No. ZR2012EEL01), and the Fundamental Research Funds for the Central Universities (No. 14CX02040A and No. 14CX06023A). The authors would like to thank Dr. Huazhou Li for helpful discussion and suggestion.

\section{References}

Anselmetti F S and Eberli G P. The velocity-deviation log: a tool to predict pore type and permeability trends in carbonate drill holes from sonic and porosity or density logs. AAPG Bulletin. 1999. 83(3): 450-466

Anselmetti F S, von Salis G A, Cunningham K J, et al. Acoustic properties of Neogene carbonates and siliciclastics from the subsurface of the Florida Keys: implications for seismic reflectivity. Marine Geology. 1997. 144(1): 9-31

Assefa S, McCann C and Sothcott J. Velocities of compressional and shear waves in limestones. Geophysical Prospecting. 2003. 51(1): $1-13$

Berryman J G. Single-scattering approximations for coefficients in Biot's equations of poroelasticity. The Journal of the Acoustical Society of America. 1992. 91(2): 551-571

Chen Y, Huang T F and Liu E R. Rock Physics. Hefei: University of Science and Technology of China Press, 2009. 56-62 (in Chinese)

Chopra S and Huffman A R. Velocity determination for pore-pressure prediction. The Leading Edge. 2006. 25(12): 1502-1515

Dutta N C. Geopressure prediction using seismic data: current status and the road ahead. Geophysics. 2002. 67(6): 2012-2041

Eberli G P, Baechle G T, Anselmetti F S, et al. Factors controlling elastic properties in carbonate sediments and rocks. The Leading Edge. 2003. 22(7): 654-660

Gassmann F. Elasticity of porous media. Viertel. der Natur. Gessellschaft in Zürich. 1951. 96: 1-23

Gutierrez M A, Braunsdor N R and Couzens B A. Calibration and ranking of pore-pressure prediction models. The Leading Edge. 2006. 25(12): 1516-1523

Kenter J A M and Ivanov M. Parameters controlling acoustic properties of carbonate and volcaniclastic sediments at site 866 and 869 Proceedings of the Ocean Drilling Program, Scientific Results. 1995. Vol. 143: 287-303

Kumar M and Han D H. Pore shape effect on elastic properties of carbonate rocks. 75th SEG Annual Meeting, 2005, Expanded Abstracts. 1477-1481
Kuster G T and Toksǒz M N. Velocity and attenuation of seismic waves in two-phase media. Geophysics. 1974. 39(5): 587-618

Liu X X, Yin X Y and Zhang F. S-wave velocity estimation method in carbonate reservoirs. Journal of China University of Petroleum. 2013. 37(1): 42-49 (in Chinese)

Ma Y S, Mei M X, Chen X B, et al. Sedimentology of Carbonate Reservoirs. Beijing: Geological Publishing House. 1999. 161-163 (in Chinese)

Sayers C M, den Boer L D, Nage Z R, et al. Well-constrained seismic estimation of pore pressure with uncertainty. The Leading Edge. 2006. 25(12):1524-1526

Sayers C M, Johnson G M and Denyer G. Predrill pore-pressure prediction using seismic data. Geophysics. 2002. 67(4): 1286-1292

Sun S Z, Wang H, Liu Z, et al. The theory and application of DEMGassmann rock physics model for complex carbonate reservoirs. The Leading Edge. 2012. 31(2): 152-158

SY/T 5431-2008. The design method of casing program. 2008 (in Chinese)

Traugott M. Pore/fracture pressure determination in deep water. World Oil. 1997. Aug: 68-70

Verwer K, Braaksma $\mathrm{H}$ and Kenter J A. Acoustic properties of carbonates: effects of rock texture and implications for fluid substitution. Geophysics. 2008. 73(2): B51-B65

Wang H, Sun S Z, Yang H, et al. The influence of pore structure on P-\&S-wave velocities in complex carbonate reservoirs with secondary storage space. Petroleum Science. 2011. 8(4): 394-405

Wang Z J. Fundamental of seismic rock physics. Geophysics. 2001. 66(2): 398-412

Wang Z J. Seismic anisotropy in sedimentary rocks, part 2: laboratory data. Geophysics. 2002. 67(5): 1423-1440

Weger R J. Quantitative pore/rock type parameters in carbonates and their relationship to velocity deviation. Ph.D. Dissertation. University of Miami. 2006, May. 216-218

Xu S and White R E. A new velocity model for clay-sand mixtures. Geophysical Prospecting. 1995. 43: 91-118

Xu S Y, Chen G L, Zhu Y P, et al. Carbonate rock physics: analytical models and validation using computational approaches and lab/log measurements. The International Petroleum Technology Conference, Dubai, U.A.E., 2007, Expanded Abstracts, IPTC 11308

Xu Y, Gardner G H F and McDonald J A. Some effects of velocity variation on AVO and its interpretation. Geophysics. 1993. 58(9): 1297-1300

Zhang $\mathrm{J}$ and Wieseneck J. Challenges and surprises of abnormal pore pressures in the shale gas formations. SPE Annual Technical Conference and Exhibition, 30 October-2 November 2011, Denver, Colorado, USA (paper SPE 145964)

(Edited by Sun Yanhua) 